\title{
La lengua de los tobas bolivianos en un vocabulario inédito del franciscano Hermán Cattunar (c. 1911)
}

The language of the Bolivian Toba in an unpublished vocabulary by Franciscan Hermán Cattunar (c. 1911)

\section{María Belén Carpio and Marcela Mendoza}

\section{(2) OpenEdition}

Electronic version

URL: https://journals.openedition.org/corpusarchivos/4800

DOI: 10.4000/corpusarchivos. 4800

ISSN: 1853-8037

Publisher

Diego Escolar

Electronic reference

María Belén Carpio y Marcela Mendoza, «La lengua de los tobas bolivianos en un vocabulario inédito del franciscano Hermán Cattunar (c. 1911)», Corpus [En línea], Vol. 11, №. 1 | 2021, Publicado el 29 junio 2021, consultado el 03 julio 2021. URL: http://journals.openedition.org/corpusarchivos/4800 ; DOI: https://doi.org/10.4000/corpusarchivos.4800

This text was automatically generated on 3 July 2021.

Licencia Creative Commons: Atribución-NoComercial 2.5 Argentina (CC BY-NC 2.5 AR) 


\section{La lengua de los tobas bolivianos en un vocabulario inédito del franciscano Hermán Cattunar (c. 1911) $)^{1}$}

The language of the Bolivian Toba in an unpublished vocabulary by Franciscan Hermán Cattunar (c. 1911)

María Belén Carpio and Marcela Mendoza

\section{EDITOR'S NOTE}

Fecha de recepción del original: 08/04/2021

Fecha de aceptación para publicación: 17/05/2021

\section{Introducción}

1 En este trabajo analizamos el contenido de un vocabulario toba atribuido al franciscano Hermán Cattunar (c. 1911), atendiendo al contexto histórico de producción del mismo. Fray Cattunar llegó a Bolivia, en 1895, junto con otros franciscanos italianos para participar en las actividades misionales del Colegio Franciscano de Tarija. Su trabajo lingüístico más conocido es el diccionario chiriguano-español y español-chiriguano realizado en colaboración con Fray Santiago Romano, publicado en Tarija en 1916. El Vocabulario toba cuyo análisis lingüístico presentamos está incluido en un conjunto de cinco libretas pertenecientes a Cattunar. Las libretas se conservan en el Archivo Franciscano del Convento Franciscano de Tarija. Además del vocabulario, contienen apuntes sobre la lengua toba y el catecismo en toba ${ }^{2}$. 
2 El trabajo está organizado de la siguiente manera: en \$2 describimos aspectos etnohistóricos sobre los grupos tobas bolivianos, particularmente durante el período en que mantuvieron relaciones más estrechas con los misioneros franciscanos, en la segunda mitad del siglo XIX; en \$3 presentamos datos biográficos sobre Fray Cattunar, con especial énfasis en su obra lingüística; en $\S 4$ nos referimos a la estructura del Vocabulario toba (Cattunar c. 1911) y mencionamos otros registros de la lengua hablada por los tobas de Bolivia a fines del siglo XIX; en §5, a partir de los datos proporcionados por Cattunar, analizamos los pronombres libres, los índices pronominales verbales, las estrategias de negación, y los números cardinales. El análisis de una variedad de toba hablada a fines del siglo XIX aporta materiales para el estudio de posibles procesos de gramaticalización entre lenguas guaycurúes, no detectables al estudiar cada lengua sincrónicamente, y proporciona datos lingüísticos -que han permanecido inéditos hasta ahora-para investigar la hipótesis de contacto histórico entre los tobas bolivianos y los tobas occidentales en Argentina.

\section{Los tobas bolivianos en la segunda mitad del siglo $\mathrm{XIX}$}

3 Históricamente, los grupos tobas vivían en las llanuras bañadas por el río Alto Pilcomayo, en la región del Chaco boreal comprendida entre los paralelos $21^{\circ} 15^{\prime} \mathrm{y}$ $23^{\circ} 26^{\prime}$ latitud sur. Ocuparon esos territorios desde tiempos prehistóricos, es decir, desde antes de la llegada de los españoles. Pequeños grupos de personas emparentadas por lazos sanguíneos, afinales y de amistad que hablaban una lengua luego clasificada como guaycurú, se movilizaban estacionalmente por sus territorios tradicionales durante el ciclo anual. Thouar (1906, p. 23) estimó que estos pequeños grupos móviles reunían unas 40 o 50 personas-estimación que coincide con cálculos efectuados, a partir de datos de diversos autores, por Mendoza (2019c).

4 Los pequeños grupos estaban liderados por un jefe y mantenían entre sí relaciones solidarias, aunque a veces también contenciosas, comunicándose y visitándose frecuentemente. Además, se vinculaban con grupos étnicos vecinos que hablaban otras lenguas (Mendoza 2019b). Los tobas se autodenominaban komelike en la grafía de Thouar (1891, p. 420); komlék si se referían a una sola persona, en la grafía de Karsten (1993[1923], p. 125). Sus vecinos avá-guaraní o "chiriguanos" los llamaban toba y este es el nombre adoptado por los europeos para referirse a ellos. Desde mediados del siglo $\mathrm{XVI}$, los komelike del Alto Pilcomayo aparecen en los documentos coloniales como tobas (Mendoza 2019a).

5 Los pequeños grupos familiares, que en otros trabajos hemos denominado "bandas", demostraron una extraordinaria cohesión interna durante el período colonial. Tenían la capacidad de coaligarse prontamente para enfrentar enemigos comunes. Los varones adultos, principalmente aquellos que habían sido iniciados como guerreros, efectuaban raides ofensivo-defensivos a grandes distancias y regresaban a sus aldeas con bienes y trofeos, que a veces incluían escalpes y cautivos. Esa cohesión social les permitió hostigar con frecuencia a las aldeas avá-guaraní y contener el avance de los pobladores coloniales por cientos de años.

6 Sin embargo, desconocemos cómo los pequeños grupos familiares se identificaban a sí mismos y cómo definían sus territorios. Ocasionalmente, encontramos referencias de 
los franciscanos a grupos familiares identificados por el nombre de un líder, por ejemplo, los "cusaireta" o "la gente de Cusarai" (Giannecchini 1988[1879], p. 327). Karsten (1993[1923], p. 20) se refiere a "familias particulares" que se movilizaban por distintos lugares. Sospechamos que las bandas o grupos familiares de los tobas del Alto Pilcomayo tenían identidad propia y territorios que ocupaban habitualmente, tal como ocurría entre otros tobas occidentales. Es posible también que algunas bandas con territorios colindantes, que mantenían entre sí relaciones sociales más próximas, hubiesen formado lo que entre otros tobas occidentales se ha identificado como "grupos regionales"; aunque los escritos de los misioneros solo permiten distinguir un grupo coaligado numeroso, llamado "tobas del este"-aquellos que permanecieron afiliados a la misión San Francisco, quienes se relacionaban con frecuencia con los aváguaraní de las misiones de Tarairí y Machareti- y otro grupo coaligado llamado "tobas del sur", cuyos líderes tuvieron relaciones menos amistosas con los franciscanos y parecen haberse apoyado más en los vecinos weenhayek, guisnay, y tapiete.

7 Para apropiarse de los recursos naturales, los grupos familiares móviles utilizaban un modelo de adaptación estacional al ambiente basado en la caza, pesca y recolección. Este modelo socioambiental, modificado y adecuado a las circunstancias, mantuvo su viabilidad durante mucho tiempo, aún después de que los grupos guaycurú-hablantes iniciasen interacciones constantes, productivas y también agresivas con los pobladores bolivianos que gradualmente fueron ocupando la región (Mendoza 2019c, 2020).

8 A mediados del 1800, los hacendados y vaqueros de los alrededores de Tarija y Caiza comenzaron a expandirse sobre los territorios de los pueblos indígenas de la llanura chaqueña. Hasta entonces la frontera chaqueña había quedado casi estacionaria, aunque con frecuentes incursiones tanto por los tobas aliados con otros guerreros o por los soldados y milicianos bolivianos. El ímpetu expansivo de los bolivianos podría atribuirse, según Langer y Bass Werner de Ruiz (1988), a (i) la revitalización de la economía de la plata y el aumento de la demanda de ganado en los centros mineros, (ii) la creciente consolidación del poder político y militar del estado boliviano, y (iii) la introducción de nuevas armas de fuego por los colonos.

9 Según Schmieder (1926, p. 146), la penetración fue iniciada por colonos, vaqueros y comerciantes de áreas vecinas que avanzaron paso a paso hacia el interior de la llanura. El estado nacional estableció puestos militares para proteger a los pobladores y realizar expediciones punitivas contra los guerreros que organizaban raides contra las estancias y los poblados. A su vez, alentó a los misioneros franciscanos para que estableciesen misiones donde existían numerosas poblaciones indígenas. Al expandirse las haciendas ganaderas, adquirieron relevancia los pequeños comerciantes-vinculados con las grandes casas de comerciantes de Tarija-que comerciaban bienes y ganado no solo con los puesteros sino también con las comunidades indígenas (Langer y Hames 1994, p. 299).

10 La segunda mitad del 1800 , entonces, fue un período marcado por violencia y acomodación. Autores contemporáneos hablaron de guerras continuas (Brettes 1890; Colocci 1908). Ataques sorpresa de los tobas, cuatrerismo, contraataques de las milicias, robo de niños y niñas indígenas por los milicianos, cautiverio y trabajo forzado de hombres y mujeres. Por ejemplo, entre julio de 1847 y enero de 1859 en la parroquia de Caiza se registró la muerte de 31 personas víctimas del "indomable furor" de los tobas (Corrado 1884, pp. 399-400) y Thouar (1906, p. 23) dijo que los tobas asesinaron más de 50 hombres entre 1882 y 1900. Sin embargo, hay muchos otros registros de violencia. 
Varios documentos indican que algunas acciones de los soldados bolivianos fueron más "salvajes" que las de los indígenas (Langer y Bass Werner de Ruiz 1988, p. 285). En las fiestas patrióticas y religiosas bolivianas, autores contemporáneos observaron que participaban jinetes vestidos de tobas, adornados con plumas de avestruz, para representar a los típicos "salvajes" (Reclus 1894, p. 876; Weddell 1851, p. 266).

Durante este período, por lo menos tres generaciones de hombres y mujeres tobas trataron de negociar un modo de integración al estado boliviano, manteniendo cierto control sobre sus territorios. Calzavarini (2006c, p. 37) comenta sobre la integración de los jóvenes en la sociedad boliviana y la pérdida de poder político sobre sus comunidades. Algunas familias se acercaron y permanecieron en la misión San Francisco, otras se emplearon en las haciendas de los alrededores de Tarija y en los ingenios de Argentina (Wright 1907, p. 448).

En la década del 1880, como alternativa a las misiones, el gobierno concibió la colonización militar por empresa privada, autorizando a fundar pequeñas colonias con fortines. Cada colono que se asentaba en los fortines recibía una legua cuadrada de terreno en propiedad. Según Langer y Bass Werner de Ruiz (1988, p. XIX), "los ganaderos eran enemigos de los tobas, porque les comían las vacas o las vendían ... siguió [entonces] una verdadera masacre de indios. Los que no se presentaban a trabajar eran vistos como enemigos de los ganaderos y los mataban indiscriminadamente".

El tratado de paz celebrado en San Francisco el 15 de septiembre de 1884 entre quince líderes tobas, tres líderes noctenes-weenhayek, dos líderes tapietes, tres líderes chorotes y militares bolivianos registra una de las pocas ocasiones en que los documentos preservan la voz de los líderes indígenas. Colectivamente expresaron lo siguiente:

Cierren ustedes todos los caminos de la guerra como lo hemos hecho nosotros y si encontramos en alguna parte sangre, la cubriremos con tierra para que no se conserve ningún recuerdo. Nos hemos inferido grandes males de parte a parte y como ahora ya somos amigos no tenemos que hacernos cargos ni reclamos de ningún género porque si es verdad que nosotros hemos robado ganado vacuno, caballar y mular y muerto caraís y hecho cautivas algunas señoras, las que siempre hemos devuelto, ustedes nos han quitado también caballos y mulas, nos han muerto mayor número de los nuestros, nos han hecho cautivos a nuestras mujeres e hijos en número infinito, jamás nos han devuelto a nadie, así es que de lo pasado no hay para qué recordar nada (documento 668, en Langer y Bass Werner de Ruiz 1988, p. 252).

Sucedieron años de asaltos, ocupación de tierras por los pobladores bolivianos, epidemias de viruela, matrimonios mixtos entre mujeres tobas y hombres bolivianos (Chervin 1908, p. 137). Varios grupos familiares se establecieron definitivamente en la provincia argentina de Salta, donde permanecen hasta hoy.

Hacia 1830, Acide D’Orbigny $(1839$, p. 94) estimó que la población toba de Bolivia cerca de la Cordillera de los Chiriguanos habría sumado casi 6000 personas. Cardús (1884) calculó que la población toba sumaba entre 3000 y 4000 personas. El franciscano Sebastián Pifferi (1895, p. 24) indicó que esta población no excedería de 4000. En 1912, Karsten (1993 [1923]) estimó que los tobas de Bolivia eran unas 1500 personas. En 2012, el censo de población de Bolivia (INE 2015, p. 29, gráfico 17) enumeró 86 personas tobas, la mayoría establecida en un área rural del Departamento de Tarija (INE 2015, cuadro 11 y 12). 


\section{Fray Hermán Cattunar}

Hermán Cattunar nació en Capo d'Istria, Italia. El franciscano fue reclutado para el trabajo misionero durante una visita del fraile Sebastián Pifferi a la Provincia Franciscana de Roma (Calzavarini 2006b, pp. 108-109). Pifferi había viajado a Roma en diciembre de 1894 para dar a conocer a los Superiores de la Orden el estado de los Colegios, Hospicios y Misiones de Bolivia (Paz, 1913, p. 88). Cuando regresó a Bolivia en 1895, lo acompañaron Cattunar y otros franciscanos italianos que iban a participar en las actividades misionales del Colegio Franciscano de Tarija.

En 1916, el franciscano Pedro Corvera (1998) compiló un catálogo del Archivo Franciscano. Esta compilación incluye entre los manuscritos e impresos de los misioneros, un cuaderno con apuntes sobre la lengua toba perteneciente al Prefecto de las Misiones José Gianelli (documento 694, Corvera 1998, p. 65) y otros documentos clasificados como "miscelánea de apuntes, de cuentas, de términos chiriguanos y de términos tobas”, también de autoría de Gianelli (documento 700, Corvera 1998, p. 65). El franciscano Gerardo Maldini agregó luego una adenda al catálogo de Corvera, donde se registran varios apuntes de Hermán Cattunar (documento 1087, Corvera 1998, p. 92).

8 Recientemente, Combès y Oliva (2020) elaboraron una lista exhaustiva de los documentos que se encuentran en el archivo y examinaron las condiciones de su producción. En general, se trata de un corpus en su mayor parte inédito, consistente en más de 6.000 folios fechados entre 1771 y 1923. Los documentos contienen los primeros registros lingüísticos del toba boliviano, wichí (vejóz y noctén-weenhayek) y guaraníchiriguano (avá y simba), lenguas habladas por comunidades indígenas que participaron en las misiones franciscanas de Bolivia hasta comienzos del siglo XX.

9 Según CombÈs y Oliva (2020), la obra de Cattunar que se conserva en el archivo (Tabla 1) consiste en once ítems, fechados entre 1900 y 1923. Los autores atribuyeron a Cattunar el "Catecismo en noctén y chiriguano" y las "Palabras en noctén", fechados en 1900. Los ítems restantes son de autoría de Cattunar, uno de ellos en colaboración con Fray Inocencio Massei. 


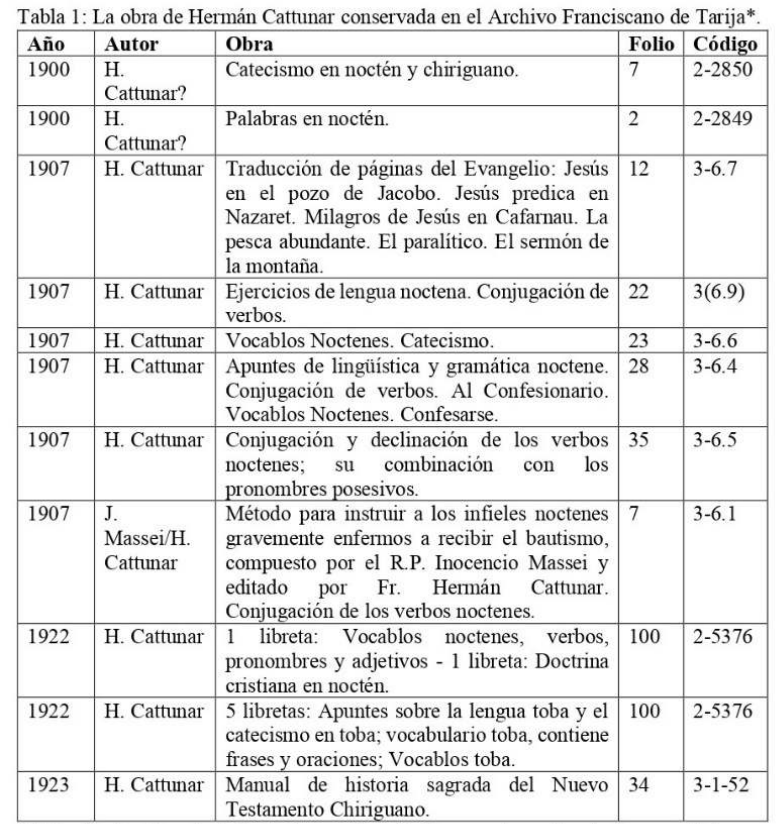

*Nota: Contenido adaptado de los cuadros 1: Documentos sobre la lengua chiriguana conservados en el Archivo Franciscano de Tarija (s. XIX-XX), 2: Documentos sobre la lengua wichí conservados en el mismo archivo (s. XIX-XX) y 3: Documentos sobre la lengua de los tobas bolivianos conservados en el archivo (s. XIX-XX) en Combes \& Oliva (2020).

Tabla $N^{\circ} 1$

El trabajo lingüístico más conocido de Cattunar es el diccionario chiriguano-español y español-chiriguano, publicado en Tarija en 1916. Fue confeccionado teniendo a la vista diversos manuscritos de misioneros del apostólico colegio de Santa María de los Ángeles de Tarija y particularmente el Diccionario Chiriguano etimológico del Prefecto de las Misiones Doroteo Giannecchini. Giannecchini había comenzado a escribir el diccionario chiriguano “... por indicación del Padre Pifferi, Perfecto de Tarija en 1885 ...; lo prosiguieron los Padres Romano y Puccelli y últimamente el Padre Cattunar lo editó como obra de los Padres Romano-Cattunar" (Barrado Manzano 1945, p. 78). El Prefecto Santiago Romano, coautor del diccionario, estaba familiarizado con la lengua hablada por los avá-guaraníes que vivían en las misiones ${ }^{3}$.

21 La publicación del diccionario chiriguano-español y español-chiriguano de Romano y Cattunar fue prontamente listada en Acta Ordinis Fratrum Minorum (Cimino 1917), e inmediatamente despertó el interés de lingüistas y etnógrafos interesados en la hasta entonces poco conocida lengua y cultura "chiriguana". Entre los autores que citan el diccionario de Romano y Cattunar figuran Erland Nordenskiöld (1922), Paul Rivet (1924), A. Meilleit y Marcel Cohen (1924), Alfred Métraux (1948), y J. Alden Mason (1950). Destacando la importancia de este diccionario bilingüe, Roberto LehmannNitsche (1924-1925, p. 80) dijo que las informaciones de índole general y etnográfica disponibles a comienzos del siglo XX sobre los guaraní-chiriguanos, “... se deben a las investigaciones del Padre Doménico de la Campana, del Barón Erland Nordenskiöld y de Fray Bernardino Nino; el idioma chiriguano es conocido gracias al celo de varios misioneros franciscanos, ante todo los Padres Santiago Romano y Hermán Cattunar".

Dado que la producción más prolífica de Cattunar está fechada a comienzos del 1900, es posible que el Vocabulario Toba que analizamos aquí haya sido compilado varios años antes de la fecha en que se catalogaron las libretas. En 1911, por ejemplo, Cattunar 
certificó un interesante mapa del Chaco boliviano realizado por J. García Meza (reproducido por Calzavarini 2006c, p. 37 y CombÈs 2019, fig. 2). Como ocurrió con la elaboración del diccionario chiriguano, es posible que Cattunar haya utilizado manuscritos sobre la lengua toba elaborados por otros misioneros, particularmente Gianelli (referido más arriba, en Corvera 1998) y Giannecchini (1996 [1898]), ambos familiarizados con la lengua y la cultura toba. Se conservan muchos textos que atestiguan la relación cordial que existió entre Gianelli y la primera generación de hombres y mujeres tobas que vivieron en la Misión San Francisco Solano (por ejemplo, Corrado 1861; Langer y Bass Werner de Ruiz 1988, pp. 289-303).

Romano (coautor con Cattunar del diccionario chiriguano) también conocía la lengua toba. Romano fue encargado en 1906 de entregar las Misiones San Francisco Solano (originalmente destinada a los tobas del alto Pilcomayo) y San Antonio de Padua (originalmente destinada a los noctén-weenhayek del alto Pilcomayo) a las autoridades bolivianas (Calzavarini Ghinello 2006d). Mientras estuvo a cargo de las misiones sobre el Alto Pilcomayo, Romano interactuó frecuentemente con gente toba. Por ejemplo, el 14 de marzo de 1906 recibió en la misión San Francisco la visita del jefe toba Taicolik con otros cuatro hombres. Taicolik le comunicó su disgusto con las decisiones tomadas por autoridades bolivianas y dijo que planeaba ir a trabajar con su gente a la Argentina (Calzavarini Ghinello 2006d, p. 912; Combès 2019b). En agosto de 1906, algunos hombres tobas "del norte del Pilcomayo" que estaban trabajando en el ingenio azucarero San Pedro de Jujuy accedieron a participar en un estudio de antropología física realizado por Roberto Lehmann-Nitsche, en colaboración con el fotógrafo Carlos Bruch. El antropólogo utilizó una metodología comúnmente empleada a comienzos del siglo XX que incluía mediciones corporales. Durante esta investigación, Pirayú, Sirán, Huaki, Sükorii, Pegro y el cacique Kotaiki fueron medidos y fotografiados. Los nombres indígenas aparecen según la grafía empleada por Lehman-Nitsche, que agregó "sic!" a continuación del nombre de Pegro (fotos de Huaiki y Sirán por Carlos Bruch, en Lehman-Nitsche (1908, Plancha XLII), foto de Pirayú por Carlos Bruch, en LehmanNitsche (1908, Plancha XLIVI).

Estimamos que los hombres tobas que participaron en el estudio de Lehman-Nitsche podrían haber sido hablantes nativos de la lengua documentada en el vocabulario de Cattunar. Nos basamos en las siguientes razones: (a) desde fines del 1800s, los tobas de Bolivia migraban estacionalmente a los ingenios del norte argentino, tal como lo indicaron los misioneros franciscanos y administradores del gobierno que documentaron esas migraciones laborales; (b) Kotaiki, Pirayú, Sirán, Huaiki, Sükorii y Pegro son las únicas personas identificadas como tobas provenientes "del norte del Pilcomayo", otros tobas fueron identificados como provenientes "del Pilcomayo" o "abajeños"; (c) el cacique Kotaiki, con una edad aproximada de 35 años según estimación del antropólogo, posiblemente haya sido miembro del grupo familiar del antiguo cacique Cotaique, quien es uno de los firmantes del tratado de paz del 1 de noviembre de 1859. Este tratado fue firmado en la Misión Tarairí entre líderes tobas del Alto Pilcomayo y líderes militares del Distrito de Salinas, de la región chaqueña de Tarija. En este tratado también participaron los franciscanos Alejandro María Corrado y José Gianelli (Cardús 1886; Corrado 1884; Langer y Bass Werner de Ruiz 1988), misioneros vinculados a la fundación de San Francisco Solano, iniciada por Corrado en 1860. 


\section{Registros de la lengua hablada por los tobas de Bolivia a fines del siglo XIX}

El Vocabulario toba (Cattunar c. 1911) consiste en 34 hojas, escritas de puño y letra en el anverso y reverso. Posee un número por hoja en el extremo superior derecho. ${ }^{4}$ Generalmente, se presenta el paradigma de persona y número de los verbos y nombres poseídos, y, en ocasiones, se incluyen oraciones. Para realizar el análisis, elaboramos una base de datos que permite realizar búsquedas desde la lengua indígena, desde el español, y según persona y número. En total, resultaron 335 entradas, de las cuales 126 poseen como núcleo un verbo. No se explicita el contexto de elicitación, pero es posible hipotetizar, a partir del tipo de verbos que se registraron y por la mención a la oración católica denominada "Padre Nuestro" ("Padre n.") en la página [33'], que ésta pudo funcionar como uno de los disparadores de la elicitación.

Como dijimos más arriba, es posible que el Vocabulario toba que analizamos aquí haya sido compilado varios años antes de la fecha en que se catalogaron las libretas de Cattunar y podría incluir vocablos registrados por Gianelli, e incluso palabras registradas por Giannecchini y conocidas por Romano. De modo que, probablemente la lengua que analizamos en este trabajo represente la manera en que hablaban los tobas de Bolivia en la segunda mitad del siglo XIX, el período de la historia de este pueblo en el que contamos con la mayor cantidad de referencias bibliográficas para entender el devenir de su sociedad.

Otros autores registraron vocablos de la lengua hablada por los tobas bolivianos entre mediados del siglo XIX y comienzos del siglo XX. Por ejemplo, Hugh Agamenon Weddell (1851, p. 328) se comunicó con los tobas del Alto Pilcomayo por medio de un intérprete y dijo que cada palabra que pronunciaban parecía costarles un sufrimiento, que su habla contenía sílabas que eran "profundos gemidos". Para dar una idea de la frecuencia con la que aparecían determinados sonidos en la lengua toba y para mostrar "lo pobre y complicado que es este lenguaje", Weddell (1851, p. 329) presentó una tabla comparando las palabras utilizadas para designar números entre los tobas, guaraníes, aymaras y quichuas. Weddell dijo que había tomado esas palabras de un texto publicado en 1836 por Pedro de Angelis (1836-1837). Sin embargo, luego de estudiar los textos publicados por de Angelis, pensamos que las referencias a la lengua toba en la colección citada por Weddell (1851, p. 141), no corresponden a la lengua hablada por aquellos que vivían en el Alto Pilcomayo sino a grupos tobas de otras regiones, posiblemente cerca de Salta y del río Bermejo.

Arthur Thouar (1891, pp. 419-421), que exploró el Chaco boliviano entre 1883 y 1888 interactuando con los tobas del Alto Pilcomayo, presenta un apéndice con notas de campo sobre la lengua hablada por estos grupos. Thouar describe características de la sociedad toba en la década de 1880 , aunque algunas partes de sus relatos han sido cuestionadas (Combès 2017). En sus respectivos estudios de la familia lingüística guaycurú, Daniel Brinton (1898, p. 184) y Lucien Adam (1899) reproducen algunas palabras en toba recogidas por Thouar. Theodor Koch-Grünberg (1902, p. 109) hizo un análisis lingüístico basado en varios autores y concluyó que, excepto por pequeñas variaciones dialectales, la lengua toba presenta rasgos similares, ya sea en el Chaco boliviano, argentino o paraguayo. 
El franciscano José Cardús $(1886$, p. 321) publicó un vocabulario toba, presuntamente también obtenido en el Alto Pilcomayo y dice que el modo de hablar de los tobas es imperioso y altivo, y que su lengua parece inventada a propósito para corresponder con el carácter serio y arrogante de los hablantes. El etnógrafo Rafael Karsten (1993 [1923], pp. 121-134) realizó trabajo de campo con los tobas bolivianos en 1911-1912 y publicó la etnografía más conocida sobre este pueblo, que incluye un vocabulario.

\section{Análisis de los datos lingüísticos}

30 A partir de los datos proporcionados en el Vocabulario Toba, presentamos, desde un enfoque funcionalista, los sistemas pronominales, las estrategias de negación, y los números cardinales.

\subsection{Sistemas pronominales}

De acuerdo con Cysouw $(1998,2003)$, los sistemas pronominales están compuestos por signos lingüísticos especializados en la codificación de deixis de persona. Los elementos pronominales pueden ser clasificados, según el autor, en dos dimensiones: i. sintagmática: en función de su interacción con otros morfemas en la lengua; de modo que los morfemas con el mismo estatus sintagmático constituyen un sistema pronominal, y ii. paradigmática: que contempla la variación dentro de cada sistema pronominal. A los fines de sistematizar los datos del Vocabulario, se tomaron en cuenta ambas dimensiones. Específicamente, la dimensión sintagmática nos permitió distinguir: pronombres libres e índices pronominales verbales según su función sintáctica $\mathrm{S}$, argumento único de una cláusula intransitiva, A o $\mathrm{P}$, argumento más parecido al agente o al paciente de una cláusula transitiva, y $\mathrm{T}$ o $\mathrm{R}$, argumento más parecido al tema o al receptor de una cláusula bitransitiva.

\subsubsection{Pronombres libres}

En el cuadro 1 mostramos los lexemas que fueron registrados como pronombres libres funcionando como S o A en el Vocabulario. En negrita resaltamos los que son incluidos por Cattunar (c. 1911) bajo el título "Pronombre personal" en el reverso de la primera hoja del Vocabulario, y, luego de las líneas punteadas, incluimos los lexemas que ocurren con muy baja frecuencia codificando un argumento $\mathrm{S}$ o A. ${ }^{5}$ 
Cuadro 1. Pronombres libres con función S/A

\begin{tabular}{|c|c|c|}
\hline \multirow[b]{3}{*}{1} & \multirow{3}{*}{ jayím (la) } & NO-SINGULAR \\
\hline & & comí (1d) \\
\hline & & $\begin{array}{l}\text { comím comi (2) } \\
\text { jechocodén (3d) }\end{array}$ \\
\hline \multirow{2}{*}{2} & \multirow[t]{2}{*}{ am (1b) } & amí (1d) \\
\hline & & amchocodén (3e) \\
\hline \multirow{3}{*}{3} & $\begin{array}{c}\text { nacaedá a } \\
(1 \mathrm{c})(8 \mathrm{a})(9 \mathrm{a})(10 \mathrm{a})\end{array}$ & $\begin{array}{c}\text { nacaedaguá } \sim \mathrm{a} \\
(7 \mathrm{~b})(11 \mathrm{~b})(12 \mathrm{~b})(13 \mathrm{~b})\end{array}$ \\
\hline & $\begin{array}{c}\text { ñacañik (5a) (6a) } \\
\text { nacaeñí i (7a) } \\
\text { nacada'm (11a) (13a) } \\
\text { cada (16a) } \\
\text { jiyim (17a) } \\
\text { jegam (14a) } \\
\text { cadami (18a) }\end{array}$ & $\begin{array}{c}\text { nacaida mi (5b) } \\
\text { nácaedaguá (1f) } \\
\text { jedaam (8b) } \\
\text { cadaguá (16b) } \\
\text { id'iam (17b) } \\
\text { jedachalo (9b) } \\
\text { nacaedaga (10b) }\end{array}$ \\
\hline & \multicolumn{2}{|c|}{$\begin{array}{l}\text { nacaedá mi (12a) (18b) } \\
\text { jedam (15) }\end{array}$} \\
\hline
\end{tabular}

Cuadro $N^{0} 1$

\section{(1) a. jayim inóchiñi}

\section{'Yo bajo'}

b. am anenóchiñi anochiñi

'Tú bajas'

c. nacaedá nenóchiñi

'Él baja'

d. comí inótaañi

'Nosotros bajamos'

e. amí nenóchiñi

'Vosotros bajáis'

\section{f. nácaedaguá inochiñi}

'Ellos bajan' [10]

Se observó un ejemplo en el que el pronombre de primera persona plural se reduplica (2):

\section{(2) comím comiguá jopa yataco peso}

'Nosotros tenemos un peso' [5]

A su vez, se registró el uso de los lexemas jechocodén y am chocoden para expresar primera y segunda persona plural como pronombres libres que funcionan como argumento S de la cláusula (3); y je chogodén y am chogodén para codificar estas personas como poseedoras junto al nombre yidik 'amigo' (4).

\section{(3) a. jayim ñuvi}

'Yo vengo' [12]

b. am ñuvi

'Tú vienes' [12]

c. ñacañik ñuvi 
54

'Él viene' [12]

d. jechocodén cad'ñuvi

'Nosotros venimos' [12]

e. am chocoden jed'ñuvi

'Vosotros venís' [12]

f. nacaidaguá cad'ñuvi

'Ellos vienen' [12]

(4) a. jayim yidik

'Mi amigo' [11']

b. am yidik

'Tu amigo' [11']

c. taiquedá ${ }^{\text {yidik }}$

'Su amigo' [11']

d. je chogodén yidik

'Nuestro amigo' [11']

e. am chogodén yidik

'Vuestro amigo' [12]

f. nacaidagua yidik

'Su amigo' [12]

La mayor variación paradigmática se observó en los lexemas que funcionan como pronombres libres de tercera persona (5)-(18). Cattunar (c. 1911) traduce los pronombres libres de tercera persona en masculino singular y plural; no se mencionan ejemplos en otros géneros.

'Él acuchilla' [11']

b. nacaida mi yijok

'Ellos acuchillan' [11']

(6) a. nacañik jlotani comí Mariano

'Él nos aparta de Mariano' [11']

b. nacaedagua jotani

'Ellos apartan' [11]

(7) a. nacaeñíi ${ }^{7}$ yalat

'Él mata' [26]

b. nacaedaguá jalatak

'Ellos matan' [26]

(8) a. nacaeda 'no'copitá

'Él desea' [28]

b. jedaam 'no'copitá

'Ellos desean' [28] 
(9) a. nacaedá yovidiñi

91 'Él respeta' [24']

b. jedachalo yovi-diñi

93

'Ellos respetan' [24']

(10) a. nacaeda no-copita

95

b. nacaedaga no-copita 

funcionando como $P^{8}$. En negrita resaltamos aquellos que Cattunar (c. 1911) presenta en forma de paradigma con el significado 'a mí, a ti, ... a vosotros y chupé (sic)" [9', 10]. En lugar de incluir, en la tercera persona, el significado del pronombre P en español ('lo, la, le, a él/ella'), el misionero menciona el pronombre de tercera persona objeto chupé ${ }^{9}$. En [28'] aparece el término adete traducido como 'fiesta', el cual es otro indicio del conocimiento del guaraní por parte de los consultantes tobas que trabajaron con Cattunar y de él mismo. De acuerdo con Combès y Oliva (2020, p. 632), la práctica de traducción al guaraní se observa en varios textos religiosos, lo cual da cuenta del manejo de esta lengua por parte de los misioneros. Específicamente, mencionan, entre otros, al catecismo noctén y chiriguano, atribuido a Hermán Cattunar (AFT 2-2850), en el cual la traducción no se realiza del noctén al español sino al "chiriguano".

Cuadro 2. Pronombres libres con función $P$

\begin{tabular}{l|c|}
\multicolumn{2}{|c}{ SINGULAR } \\
\hline 1 & jayím 'a mi' (20) \\
\hline 2 & am 'a ti' (21) \\
\hline \multirow{2}{*}{3} & nacaeda (24) \\
\cline { 2 - 2 } & chiacajop \\
\hline
\end{tabular}

\begin{tabular}{|c|}
\hline NO-SINGULAR \\
\hline comí (22) \\
\hline amí 'a vosotros' (23) \\
\hline nacaidagua (25) \\
\hline ñim 'chupé' \\
\hline
\end{tabular}

Cuadro $\mathrm{N}^{\circ} 2$ 

pronombres libres que funcionan como $R$ son los mismos que ocurren en función S, A y P. 
Tabla 2. Verbos según transitividad y flexión de persona y número

\begin{tabular}{|c|l|}
\hline $\begin{array}{l}\text { Total de verbos: } 126(100 \%) . \\
\text { Verbos semánticamente intransitivos: } 19,05 \% \\
\text { Verbos semánticamente transitivos: } \mathbf{8 0 . 9 5 \%}\end{array}$ & \\
\hline 1SG & $\mathbf{8 5 , 7 1 \% ( 1 0 8 )}$ \\
\hline 2SG & $68,35 \%(86)$ \\
\hline 3SG & $72,22 \%(91)$ \\
\hline 1PL & $66,66 \%(84)$ \\
\hline 2PL & $57,14 \%(72)$ \\
\hline 3PL & $56,34 \%(71)$ \\
\hline
\end{tabular}

TABLA N²

En la mayoría de los ejemplos, ocurre el pronombre libre en función S/A antepuesto al verbo. Con los datos disponibles, no podemos determinar si el uso de un co-nominal o nombre/pronombre libre con el mismo rol y referencia que el índice verbal S/A es obligatorio o responde a la forma de registro del misionero. En este sentido, de acuerdo a la posibilidad y obligatoriedad de ocurrencia de un co-nominal, siguiendo lo propuesto por Haspelmath (2013), podemos hipotetizar que los índices verbales se encontrarían en un estadio intermedio entre índices con un co-nominal opcional (crossindexes) u obligatorio (gramm-indexes).

En el cuadro 3, presentamos una selección de verbos con sus respectivos paradigmas de persona y número de modo tal de mostrar los índices pronominales verbales identificables en el Vocabulario. Resaltamos, en negrita, cada afijo de persona y número con el fin de poder detectar con mayor facilidad los morfemas, aun cuando en el cuadro dicho afijo aparezca en más de una celda. Las principales diferencias, en términos de indexación, se observan en los verbos sombreados en gris. ${ }^{10}$ 
Cuadro 3. Índices pronominales verbales S/A

\begin{tabular}{|c|c|c|c|c|c|c|}
\hline \multirow[t]{2}{*}{ Verbos } & \multicolumn{3}{|c|}{ SINGULAR } & \multicolumn{3}{|c|}{ NO-SINGULAR } \\
\hline & 1 & 2 & 3 & 1 & 2 & 3 \\
\hline -oit 'hacer' & $\mathbf{j}-$ & ab- & $y-$ & $j-\ldots-a k$ & au $-\ldots-i$ & yoit'itivak \\
\hline -omachi 'oir' & $j-$ & am'achí & d- & $\mathrm{j}-\ldots-\mathrm{ac}$ & am'achi & d- \\
\hline -ñjijiguen 'levantar' & ja- & agua- & ya- & janóejeguen & agua- & ya- \\
\hline -at'ogiuén 'engañar' & $j-$ & au- & $y-$ & j-...-aca & agu- & iatogǘn \\
\hline -eléu 'morir' & j- & j- & $j$ & cad- & & \\
\hline -apaguen 'enseñar’ & j- & au- & Ø-/y- & $j-\ldots-a c$ & $\begin{array}{l}\mathbf{a}-\ldots-\mathbf{i} \\
\text { au- } \ldots \text {-i }\end{array}$ & $y-$ \\
\hline$-a y i$ 'ir' & $j-$ & a- & $\mathrm{t}-$ & $\begin{array}{l}\text { comí (m') jaa-guí } \\
\text { jagagui }\end{array}$ & aegui & ta'tii-yi \\
\hline $\begin{array}{l}\text {-najop 'ser, servir' } \\
\text { (En toba delo este de Formosa, } \\
\text { nahop significa ir alrededor } \\
\text { de'). }\end{array}$ & jo- & qo- & ø- & joncajop & co- & Ø- \\
\hline -piyinot 'creer' & ja- & aûa'- & ua'- & comi'má-piyinocot & aûa'- & \\
\hline -vitipkia 'creer' & jo- & $\mathbf{a}^{\prime} \mathbf{0}-$ & iu- & Ø?:...-ca & $a^{\prime} 0-\ldots i$ & 'i'u- \\
\hline -uarainñi 'cumplir' & j- & a- & i- & $j-$ & ami ma ua'-rainñi & i- \\
\hline -ocachía 'robar' & $j-$ & 'a- & d'- & jocacha & 'a- & n'ocachita \\
\hline -enak'decir' & $j-$ & agu- & $\varnothing-$ & $j-\ldots-a c$ & av-...i & \\
\hline -ejetim -eetin 'pagar' & $j-$ & & $y-$ & comi-li-jejetínak "Pagamos" & & \\
\hline -ñuvi 'venir' & Ø- & $\varnothing-$ & ø- & cad- & jed- & cad- \\
\hline -ajota 'cuidar' & $\varnothing-$ & & d- & & & \\
\hline -ichojodin 'amparar' & Ø- & au'- & Ø- & $\varnothing-\ldots$-ac & & Ø- \\
\hline -inóchiñì 'bajar' & ø- & an-e-a- & n-e & $\begin{array}{l}\text { Ø-inótaañi } \\
\text { n-inotaañi }\end{array}$ & n-e & ø- \\
\hline -copita 'amar' & Ø- & amo- & no- & calo- & amo- & no- \\
\hline -chogotelat 'confesar' & in- & an- & ni- & inchogotajalat & an- & nichogo(pita)telat \\
\hline -acheo 'amar' & $\tilde{\mathbf{n}}_{-}$ & an & n- & $\mathbf{n}-\ldots \ldots-\mathbf{a k}$ & na nachelié & n- \\
\hline$-k i i$ 'comer' & on- & 0 & d'- & $\mathbf{i}-\ldots-$ ak & am'im'a okii & d'- \\
\hline$-i k$ 'ir' & $\mathbf{i k}$ & am an ok & nacaeda 'm'ek & comi'm'colak & ami m okíi & nacaeda'im'eke \\
\hline -cochiá 'ayudar' & i- & al- & $a(1)-$ & & & \\
\hline -cochiyé 'ayudar' & i-cochiyé & al cochial & & comil cohielí "Te ayudamos" & & \\
\hline $\begin{array}{l}\text {-alare 'enseñar } \\
\text { (mostrar)' }\end{array}$ & d- & agu- & $\varnothing-$ & & & \\
\hline
\end{tabular}

Cuadro $N^{\circ} 3$

172 El prefijo $j(V))^{11}$ es preponderante en la primera persona singular $\mathrm{y}$, como parte del circunfijo de primera persona plural junto al morfema -ac -ak -(a)ca -ga. Otros morfemas que codifican primera persona singular y plural son $\tilde{n}^{-}$e in-, los cuales también ocurren como primer segmento del circunfijo de primera persona plural. Se observó, además, la ausencia de prefijo pronominal cuando se trata de la primera persona singular y el uso del sufijo - ac como único marcador de primera persona plural.

173 En la segunda persona singular y plural, es recurrente el uso de un prefijo compuesto

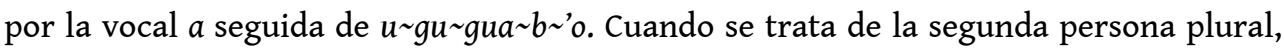
se adiciona el sufijo $-i$.

174 En la tercera persona singular y plural ocurren los prefijos $y(V)-, i-, t-, y d\left({ }^{\prime}\right)-$. El plural de tercera persona implica, por lo general, variaciones morfofonológicas en la raíz verbal.

175 En lo que respecta al argumento $P$, en el Vocabulario, se utilizan los pronombres libres (20)-(25), y solo se registraron los ejemplos (29)-(31) en los que ocurren los afijos presentados en el cuadro 4.

Cuadro 4. Morfemas que codifican al argumento $\mathrm{P}$

\begin{tabular}{l|c|}
\multicolumn{2}{c}{ SINGULAR } \\
\hline 1 & 'ta \\
\hline 2 & 'do \\
\hline 3 & $i$ ? \\
\hline
\end{tabular}

\begin{tabular}{|c|}
\hline NO-SINGULAR \\
\hline cada- $\sim$ cada \\
\hline ada- $\ldots$ - - \\
\hline$?$ \\
\hline
\end{tabular}

Cuadro $N^{\circ} 4$

En (29a-b) los morfemas 'ta 'primera persona singular P' y'do 'segunda persona singular P' parecen sufijarse al pronombre libre de tercera persona con función A nacaeda'm. En (29c-d) los morfemas cada 'primera persona plural P' y ada 'segunda persona plural P' son transcriptos por Cattunar (c. 1911) separados tanto del pronombre libre de tercera persona como del verbo. En (29d) se observa, además, un proceso de la palatalización de 
la raíz verbal, probablemente motivado por la presencia del sufijo $i-$, que junto con ada, estarían codificando segunda persona plural. En (30b)-(31b) cada- ocurre como un prefijo verbal que codifica primera persona plural, en distribución complementaria con el pronombre libre comi comí. oraciones verbales declarativas, que consisten en un único predicado con la menor cantidad posible de frases nominales y modificadores adverbiales (Payne 1985; Miestamo 2000, 2006). En cambio, siguiendo lo planteado por Veselinova (2013), las predicaciones existenciales afirmativas y negativas son estativas y postulan la presencia o ausencia de algo de un modo absoluto. En el Vocabulario, se registró el uso de la partícula negativa $\mathrm{ja}^{12}$ (32) para expresar negación junto a verbos (32b) y pronombres libres de tercera persona (33). El lexema araij aparece con el significado 'no' [18]. En (33), si bien la traducción que proporciona el misionero de nacaedá es 'sí' y ja nacaedá como 'no es', también podrían ser traducidos como '(Es) él' y 'No (es) él', respectivamente. Quizás lo mismo suceda con la traducción de nacaiñi como 'sí'.

(32) a. comi ontanrak

'Nosotros trabajamos' [25]

b. comí ja jontavirá ta ${ }^{13}$

'No debemos trabajar' [25']

(33) - noguáyaga e payak ${ }^{14} \mathrm{o}$ ?

¿¿El zorro es Dios?’

- ja nacaedá 

en el Vocabulario.

\section{Cuadro 5. Números cardinales}

\begin{tabular}{|l|l|}
\hline yátaco & 'uno' [5'] \\
\hline diácate & 'dos' [5'] \\
\hline nañi & 'tres' [5'] [24] \\
\hline latapiat & 'cuatro' [5'] \\
\hline cavage o cavaye & 'cinco' [5'] \\
\hline cavaitá & 'seis' [5'] \\
\hline yachi & 'siete' [5'] \\
\hline janti & 'ocho' [5'] \\
\hline yaguegui & 'nueve' [6] \\
\hline moichim o mvichin & 'diez' [6] \\
\hline
\end{tabular}

Cuadro $N^{\circ} 5$

Carpio (2012, pp. 82-83) describe en toba de oeste de Formosa el uso del verbo náñ ‘falta’ para codificar el cardinal ‘cinco’ y la cláusula náñi laqáya ‘falta otro’ para expresar 'tres'.

\section{Reflexiones finales}

En este trabajo analizamos datos lingüísticos referidos a los sistemas pronominales, la negación y los números cardinales extraídos del Vocabulario toba inédito de Fray Hermán Cattunar. Además, situamos el manuscrito en su contexto de producción, atendiendo tanto al trabajo de los franciscanos como a las características de sociedad de los tobas bolivianos en la segunda mitad del siglo XIX. Se trata de un análisis que posee relevancia para los estudios de la lingüística misionera franciscana, de procesos de gramaticalización y contacto lingüístico. Dado que los trabajos lingüísticos más conocidos y numerosos de los franciscanos en el Chaco boliviano se refieren a grupos 
"chiriguanos" de la cordillera y otros grupos tupí-guaraníes vecinos, el análisis del Vocabulario toba contribuye al conocimiento de la lengua que hablaban los grupos tobas de las llanuras del Río Alto Pilcomayo, cuya sociedad y cultura conocemos poco. En términos estrictamente lingüísticos, el análisis del Vocabulario toba que presentamos proporciona un valioso eslabón diacrónico para futuros estudios de procesos de gramaticalización en lenguas guaycurúes. Ofrece, además, datos concretos para profundizar el estudio de la hipótesis de contacto histórico entre los tobas de Bolivia y los llamados tobas occidentales actualmente en la provincia de Salta y en el oeste de Formosa, en Argentina.

\section{BIBLIOGRAPHY}

Adam, L. (1899). Matériaux por servir à l'etablissement d'une grammaire comparée des dialectos de la famille Guaicurú (Abipone, Mocovi, Toba, Mbaya). Paris: J. Maisonneuvre Libraire-éditeur.

Angelis, P. de (1836-1837). Colección de las obras y documentos relativos a la historia antigua y moderna de las Provincias del Río de la Plata. Tomos 5 y 6. Buenos Aires: Imprenta del Estado.

Barrado Manzano, A. (1945) Las Misiones Franciscanas en Bolivia. Sevilla: Talleres Tipográficos de la Editorial San Antonio.

Brettes, J. de (1890). Ma Mission au Chaco. Bulletin Société Languedocienne de Géographie, XIII, 64-86. Brinton, D. G. (1898). The Linguistic Cartography of the Chaco Region. Proceedings of the American Philosophical Society, 37(158), 178-205.

Calzavarini Ghinello, L. (2006a). Introducción: Parte III. Nueva presencia eclesial y franciscana en el Chaco: reducciones y parroquias a cargo de los Colegios de Tarija y Potosí. Tarija: Centro Eclesial de Documentación. (www.franciscanosdetarija.com, consultado el 21 de mayo, 2020).

Calzavarini Ghinello, L. (2006b). Introducción, Parte VIII. En Cuatro siglos de historia: la labor del P. Lorenzo Calzavarini en Presencia franciscana y formación intercultural en el sudeste de Bolivia. (pp. 95-111). Tomos IV-VII Época Republicana. Centro Eclesial de Documentación, Convento Franciscano de Tarija (www.franciscanosdetarija.com, consultado el 21 de mayo, 2020).

Calzavarini Ghinello, L. (2006c). Introducción [a los tomos IV-VII (momento republicano)]: Nueva presencia eclesial y franciscana en el Chaco: reducciones y parroquias a cargo de los Colegios de Tarija y Potosí. Parte III. (pp. 29-42). Centro Eclesial de Documentación, Convento Franciscano de Tarija(www.franciscanosdetarija.com/pag/documentos/intro_pres_franc/republica/intro_3.pdf)

Calzavarini Ghinello, L. (2006d) Diario del Padre Prefecto Santiago Romano, 1905-1907. En Presencia franciscana y formación intercultural en el sudeste de Bolivia según documentos del archivo franciscano de Tarija, 1606-1936. (pp. 897-970). Tomo V. Centro Eclesial de Documentación, Convento Franciscano de Tarija.

Cardús, J. (1886). Las misiones franciscanas entre los infieles de Bolivia: Descripción del estado de ellas en 1883 y 1884, con una noticia sobre los caminos y tribus salvajes, una muestra de varias lenguas, curiosidades de historia natural, y un mapa para servir de ilustración. Barcelona: Librería de la Inmaculada Concepción. 
Carpio, M. B. (En evaluación). Existenciales negativos en toba del oeste de Formosa. En Estrada Fernández, Z., A. y Álvarez González, A. (eds.) Dependencias Simétricas y Asimétricas: Dominios semánticos y motivaciones.

Carpio, M. B. (2012). Fonología y morfosintaxis de la lengua hablada por grupos tobas en el oeste de Formosa (Argentina). LINCOM Studies in Native American Linguistics 67. München: LINCOM Europa Academic Publisher.

Chervin, A. (1908). Anthropologie Bolivienne. Tomo I. Paris: Imprimerie Nationale.

Cimino, S. (1917). Acta Ordinis Fratrum Minorum. Vol. XXXVI, Fasc. VI, p. 142. Quarachi, Italia: Typographia Collegii S. Bonaventurae.

Colocci, A. (1908). Dal Fiume Vermiglio alla Montagna Azzurra (Rio Bermejo y Sierra Azul). Catania: C. Galàtola.

Combès, I. (2017). La mentira toba de Monsieur Thouar. Bulletin de l'Institut Français d'Etudes Andines 46(2):331-351.

Combès, I. (2019a). El delegado y sus caciques. Leocadio Trigo en el Chaco boliviano (1904-1909). Cochabamba, Bolivia: Itinerarios Editorial.

Combès, I. (2019b). Taicoliqui: Fragmentos de una vida. Boletín Americanista 79(2), 9-27.

Combès, I. (2020). Hijos del Pilcomayo. Los últimos tobas de Bolivia. Colección Scripta Autochtona. 23. (Cochabamba, Bolivia).

Combès, I. y Oliva, D. (2020). Las lenguas chaqueñas en el archivo franciscano de Tarija (Bolivia). Revista del Museo de La Plata 5(2), 618-638. DOI: 10.24215/25456377e133.

Corrado, A. M. (1861). Relación Que el Alumno de este Colegio de Tarija Alejandro Corrado Hace de la Nueva Reducción de los Tobas en 1860. Sucre: Imprenta de Beeche.

Corrado, A. (1884). Continuación de la historia del Colegio Franciscano de Tarija. En Comajuncosa, A. y Corrado, A., El Colegio franciscano de Tarija y sus misiones. Noticias históricas recogidas por dos misioneros del mismo Colegio. (pp. 279-503). Quaracchi: Tip. del Colegio de San Buenaventura.

Cysouw, M. (2003). The Paradigmatic Structure of Person Marking. Oxford University Press.

Cysouw, M. (1998). Syntagmatical Variation in the World's Pronominal Systems. En Starting, H. y Veenstra, J. (Eds.) Proceedings CLS Opening Academic Year '98/'99. Tilburg: Center for language studies, 27-50.

Dietrich, W. 2005. La primera gramática del chiriguano (Tupí-Guaraní). Rivista di Linguistica. 17(2), 347-360.

D'Orbigny, A. D. (1839). L'Homme Américain (de l'Amérique Méridionale): Considéré Sous Ses Rapports Physiologiques et Moraux. Tomo 1. Paris: Pitois Levrault.

Gianelli, J. (1988 [1863]). Relación original de las expediciones del Pilcomayo en el año 1863 y fundación de la misión San Antonio de Padua. En Langer, E. D. y Bass Werner de Ruíz, Z. Historia de Tarija, Corpus documental. (pp. 289-303). Tomo 5. Tarija: Universidad Autónoma "Juan Misael Saracho".

Giannecchini, D. (1996 [1898]). Historia natural, etnografía, geografía lingüística del Chaco boliviano 1898. Calzavarini, L. (ed). Tarija: Centro Eclesial de Documentación.

Haspelmath, M. (2013). “Argument indexing: a conceptual framework for the syntax of bound person forms”. En Bakker, D. y M., Haspelmath (eds.) Languages across boundaries: Studies in memory of Anna Siewierska (pp.197-226). Berlin: Mouton De Gruyter. 
Instituto Nacional de Estadística (INE) (2015). Censo de Población y Vivienda, 2012. Características de la Población. La Paz: Estado Plurinacional de Bolivia.

Karsten, R. (1993 [1923]). Los indios tobas del Chaco boliviano. Traducido por Daniel J. Santamaría. San Salvador de Jujuy: Universidad Nacional de Jujuy.

Koch-Grünberg, T. von (1902). Die Guaikurústämme IV. Globus LXXXI (7), 105-112.

Krivoshein de Canese, N. (1983). Gramática de la lengua guaraní. Paraguay: Edición de la autora.

Langer, E. D. y Bass Werner de Ruiz, Z. (1988). Historia de Tarija, Corpus Documental. Tomo V. Tarija, Bolivia: Universidad Autónoma Juan Misael Saracho.

Langer, E. D. y Hames, G. L. (1994). Commerce and Credit on the Periphery: Tarija Merchants, 1830-1914. The Hispanic American Historical Review 74(2), 285-31.

Lehmann-Nitsche, R. (1924-1925). La astronomía de los chiriguanos. Mitología americana VIII. Revista del Museo de La Plata 28(1):80-102.

Lehmann-Nitsche, R. (1908). Estudios antropológicos sobre los chiriguanos, chorotes, matacos y tobas (Chaco Occidental), con 50 láminas según fotografías tomadas por Carlos Bruch. Anales del Museo de La Plata Tomo 1 (entrega 2, segunda serie), 53-149.

Mason, J. A. (1950). The Languages of South American Indians. PP. 159-317. En Steward, J. H. (ed.) Handbook of South American Indians, Tomo 6, Parte 3. Smithsonian Institution Bureau of American Ethnology Bulletin 143. Washington, D.C.: Government Printing Office.

Meilleit, A. y Cohen M. (eds.) (1924). Collection Linguistique publieé par la Société de Linguistique de Paris, Tomo XVI. Paris: Libraire Ancienne Édouard Champion.

Mendoza, M. (2020). Los tobas de Bolivia: Resiliencia y adaptación en el Chaco Boreal. Revista del Museo de La Plata 5(2), 639-656.

Mendoza, M. (2019a). The Bolivian Toba (Guaicuruan) Expansion in Northern Gran Chaco. Ethnohistory 66(2), 275-300.

Mendoza, M. (2019b). Toba (Guaicuruan) hunter-gatherers in the Bolivian Chaco: Time, place, and memory. Hunter-Gatherers Research 41(1), 99-127. https://doi.org/10.3828/hgr.2018.4

Mendoza, M. (2019c). Mobile Hunter-Gatherers in Semi-Arid Lands: Toba Historical Adaptations on the Northern Gran Chaco Basin. Revista de Antropología del Museo de Entre Ríos, Argentina 5(2): 21-39. DOI 10.5281/zenodo.3703212

Métraux, A. (1948). Tribes of Mato Grosso and Eastern Bolivia. En Steward, J. H. (ed.) Handbook of South American Indians. Vol., Part 2. (pp. 465-585). Smithsonian Institution Bureau of American Ethnology Bulletin 143. Washington, D.C.: Government Printing Office.

Miestamo, M. (2000). Towards a typology of standard negation. Nordic Journal of Linguistics, 23, 65-88.

Morando, M. A. (2018). Producción misionera sobre la lingüística chiriguana. Una mirada diacrónica. Anthropos. 113(1), 151-167.

Morando, M. A. (2017). Lexicografía chiriguana en perspectiva comparada: los Guaraníes de Ruiz de Montoya y León de Santiago. Revista de Antropología del Museo de Entre Ríos. 3(1), 27-45.

Nordenskiöld, E. (1922). Deductions suggested by the geographical distribution of some post-Columbian words used by the Indians of S. America. Göteborg: Elanders boktryckeri Aktiebolag. 
Payne, J. (1985). Negation. En Shopen, T. (ed.) Language typology and syntactic description. Vol I. Clause structure. (pp. 197-242). Cambridge: Cambridge University Press.

Paz, L. (1913). Fray Sebastián F. Pifferi, ilustrísimo arzobispo de La Plata. Sucre, Bolivia: Escuela Tip. Salesiana.

Pifferi, S. (1895). Diario de la visita a todas las Misiones existentes en la República de Bolivia, escrito por su secretario y compañero de viaje Padre Zacarías Ducci. Asís, Convento de Santa María de los Ángeles: Tipográfica de la Porciúncula.

Reclus, É. (1894). South America: The Andes Regions. Parte I. En Keane, A. H. (ed.) The earth and its inhabitants. Vol. 18. New York, D. Appleton and Company.

Rivet, P. (1924). Les Indiennes Canoeiros. Journal of the Société des Americanistes de Paris vol. XVI, 169-181.

Romano, S. y Cattunar, H. (1916) Diccionario chiriguano-español y español-chiriguano: compilado teniendo a la vista diversos manuscritos de antiguos misioneros del apostólico colegio de Santa Maria de los Ángeles de Tarija y particularmente el Diccionario Chiriguano etimológico del RP Doroteo Giannecchini. Tarija: Librería Antoniana.

Schmieder, O. (1926) The East of Bolivian Andes South of the Rio Grande or Guapay. University of California Publications in Geography, 2(5), 85-210.

Thouar, A. (1891). Explorations dan's l'Amerique du Sud. Paris: Librairie Hachette.

Thouar, A. (1906). Sur le bords du Pilcomayo. Les Indiens Tobas. Journal des Voyages et des Aventures de Terre et de Mer, 523, 22-25.

Veselinova, L. N. (2013). Negative existentials: A cross-linguistic study. Rivista di Linguistica, 25(1), 107-145.

Weddell, H. A. (1851). Voyage dans le Sud de la Bolivie. En Expédition dans les Parties Centrales de l'Amérique du Sud, de Rio de Janeiro à Lima, et de Lima au Paru, 1843-1847, dirigida por Francis de Castelnau. Parte 1, Tomo 6. Paris: L. Martinet.

Weddell, H. A. (2018) Viaje en el sur de Bolivia (1845-1846). Introducción, notas y traducción del francés de Isabelle CombÈs. Colección Ciencias Sociales e Historia 45, El País: Santa Cruz de la Sierra, Bolivia.

Wright, M. R. (1907). Bolivia, el camino central de Sur-América, una tierra de ricos recursos y de variado interés. Philadelphia: George Barrie \& Sons.

\section{Fuente inédita}

Cattunar, H. (c. 1911). Vocabulario Toba. Manuscrito inédito. Archivo Franciscano de Tarija. Bolivia.

\section{NOTES}

1. Una versión anterior de este artículo será presentada en formato ponencia en el XVII Congreso de la Sociedad Argentina de Estudios Lingüísticos (SAEL) y I Congreso internacional SAEL en línea, 17-21 de mayo de 2021, Universidad Nacional de Tucumán.

2. Tuvimos acceso a una copia de este vocabulario gracias a la generosidad de Isabelle Combès y Diego Oliva. 
3. Para trabajos sobre lingüística misionera franciscana referidos al "chiriguano" ver Dietrich (2005) y Morando $(2018,2017)$, entre otros.

4. A los fines de facilitar la localización de los ejemplos, enumeramos con el número incluido por el autor el anverso de la hoja, y agregamos (') a dicho número para identificar el reverso de la misma. Los números de páginas del manuscrito son incluidos, en este trabajo, entre corchetes.

5. Mantenemos la notación utilizada por Cattunar (c. 1911). El misionero no describe la equivalencia fonética de los grafemas que utiliza, pero al conmutar y, por comparación con la variedad de toba hablada actualmente en el oeste de Formosa, es posible detectar que la letra $j$ corresponde a la consonante fricativa glotal [h] por oposición a la semiconsonante palatal y.

6. Taiquedá no ocurre en el Vocabulario como pronombre libre de tercera persona singular.

7. Nacaiñi también aparece en el Vocabulario con el significado de 'sí' [6']; lo mismo sucede con nacaedá [13] (33) (Véase \$5.2).

8. Si bien chiacajop y ñim son listados, por Cattunar (c. 1911), en el paradigma de los pronombres con función P, no se encuentran ejemplos de uso de los mismos en el Vocabulario.

9. En guaraní paraguayo, por ejemplo, los pronombres de tercera persona singular y plural como objeto directo o indirecto “...son chupe o ichupe indistintamente, van después del verbo, no son sufijos. En el plural pueden ir o no con el sufijo kuéra que significa pluralidad" (Krivoshein de Canese 1983, pp. 55-57).

10. Las consonantes b, v, c, k y q son incluidas tal como aparecen en el Vocabulario.

11. Este prefijo es isomórfico con el que codifica primera persona singular S/A y compone el circunfijo de 'primera persona grupo' en toba del oeste de Formosa (Carpio 2012, 2014).

12. Esta partícula está muy próxima fonológica y morfológicamente al clítico de negación estándar registrado en toba del oeste de Formosa (ha?) (Carpio 2012, pp. 175-177, en evaluación).

13. Ta es descripta por Cattunar [25'] como "cuidado, no (partícula posp.) (sic.) de negación para el imperativo".

14. Los tobas del oeste de Formosa utilizan actualmente la palabra payák para referirse al 'diablo'.

\section{ABSTRACTS}

Much of what we know about the society of Toba peoples in the Bolivian Chaco comes from publications of Franciscan missionaries who interacted with them in the 19th century. European explorers and ethnographers published short ethnographic descriptions and wrote down some words in Toba language, but those are insufficient for analyzing their language. Lack of linguistic data prevents us from corroborating our presumption about cultural and social ties that historically would have linked the Bolivian Tobas with the so-called western Tobas of Argentina. In this work, we systematize linguistic data in an unpublished Toba vocabulary attributed to the Franciscan Hermán Cattunar (c. 1911), considering the context in which it was produced. We present the structure of the vocabulary and describe, from a functionalist perspective, the free pronouns, the verbal pronominal indexes (Haspelmath 2013; Cysouw 2003), the negation strategies (Miestamo 2000, 2006), and the cardinal numbers as recorded by Cattunar. Our analysis contributes to understand possible grammaticalization processes in Guaycuruan languages, undetectable when each language is analyzed synchronically. It also provides materials to investigate the hypothesis of contact between the Bolivian Tobas and other Tobas in the region. 
Mucho de lo que sabemos sobre la sociedad de los tobas del Chaco boliviano se encuentra en publicaciones de misioneros franciscanos que interactuaron con ellos en el siglo XIX. Exploradores y etnógrafos europeos publicaron breves descripciones etnográficas y anotaron palabras sueltas en lengua toba que resultan insuficientes para analizar su lengua. La falta de datos lingüísticos impide corroborar nuestra presunción acerca de vínculos culturales y sociales que históricamente habrían vinculado a los tobas bolivianos con los llamados tobas occidentales de Argentina. En este trabajo, sistematizamos los datos lingüísticos de un vocabulario toba inédito atribuido al franciscano Hermán Cattunar (c. 1911), atendiendo al contexto histórico de su producción. Presentamos la estructura del vocabulario y describimos, desde una perspectiva funcionalista, los pronombres libres, los índices pronominales verbales (Haspelmath 2013; Cysouw 2003), las estrategias de negación (Miestamo 2000, 2006), y los números cardinales registrados por Cattunar. Nuestro análisis contribuye al estudio de posibles procesos de gramaticalización entre lenguas guaycurúes, no detectables al estudiar cada lengua sincrónicamente, y proporciona materiales para investigar la hipótesis de contacto entre los tobas bolivianos y otros tobas de la región.

\section{INDEX}

Palabras claves: Lingüística misionera, tobas bolivianos, sistemas pronominales, negación, números cardinales

Keywords: Missionary linguistics, bolivian Tobas, pronominal systems, negation, cardinal numbers.

\section{AUTHORS}

\section{MARÍA BELÉN CARPIO}

Instituto de Investigaciones Geohistóricas

Consejo Nacional de Investigaciones Científicas y Técnicas

Universidad Nacional del Nordeste

Argentina

Correo electrónico: belencarpio@conicet.gov.ar

\section{MARCELA MENDOZA}

Global and International Studies

Western Michigan University

Estados Unidos

Correo electrónico: marcela.mendoza@wmich.edu 